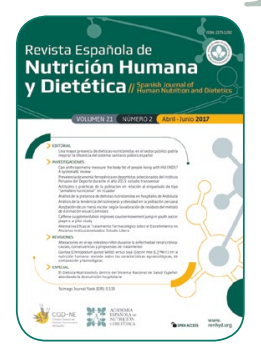

\title{
ESPECIAL
}

\section{El Dietista-Nutricionista dentro del Sistema Nacional de Salud Español: abordando la desnutrición hospitalaria}

\author{
Néstor Benítez Brito ${ }^{\mathrm{a}, *}$

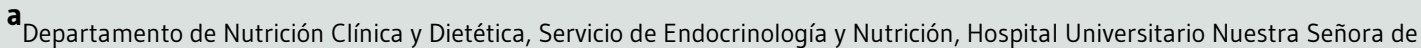 \\ Candelaria, Santa Cruz de Tenerife, España. \\ *nestorgi@hotmail.com
}

Recibido el 6 de junio de 2016; aceptado el 26 de septiembre de 2016; publicado el 17 de mayo de 2017.

\section{PALABRAS CLAVE \\ Dietista-Nutricionista; \\ Sistema sanitario; \\ Personal sanitario; \\ Desnutrición; \\ Hospitales.}

El Dietista-Nutricionista dentro del Sistema Nacional de Salud Español: abordando la desnutrición hospitalaria

\section{RESUMEN}

En España, se considera al Dietista-Nutricionista un profesional sanitario según la Ley 44/2003 de Ordenación de las Profesiones Sanitarias. No obstante, estos profesionales han encontrado serios problemas para ejercer su actividad en el ámbito sanitario, especialmente en el medio hospitalario. Por otra parte, en el año 2003, la Unión Europea desarrolló en el marco hospitalario a través del Consejo de Europa la necesidad de una atención nutricional de manera obligatoria. Entre las medidas más destacadas se encuentra la identificación y prevención de las causas de la desnutrición, junto con la búsqueda de una formación integral del Dietista-Nutricionista para tener un mejor abordaje en la atención y el soporte nutricional. A pesar de estas directrices, España es el único país de la Unión Europea que no cuenta con la presencia de este profesional dentro de su Sistema Nacional de Salud en la mayoría de Comunidades Autónomas. Por ello, la incorporación del profesional Dietista-Nutricionista a la red hospitalaria española contribuiría aún más en la detección, evaluación y tratamiento de la desnutrición, mejoraría la relación coste-efectividad de los tratamientos, aumentaría la calidad de vida de los pacientes y lograría establecer una asistencia sanitaria pública en condiciones de igualdad efectiva. 
The Dietitian-Nutritionist within the Spanish National Health System: facing hospital malnutrition

\section{KEYWORDS}

Nutritionists;

Health Services;

Health Manpower;

Malnutrition;

Hospitals.

\section{ABSTRACT}

Dietitian-Nutritionist is considered a health professional in Spain according to the Law 44/2003 on the Organization of Health Professions. However, these professionals have come across some problems in order to exercise their activity in the health sector, especially in hospitals. On the other hand, the European Union developed the necessity of a nutritional care on a mandatory basis in hospitals, through the Council of Europe in 2003. Among the most relevant measures are those to identify and prevent the causes of malnutrition and the searching of a comprehensive training for the Dietitian-Nutritionist to have a better approach in the nutritional attention and support. Despite these guidelines, Spain is the only country within the European Union that does not have incorporated these professionals in their health services in the most of Autonomous Communities. Thus, the inclusion of professional Dietitian-Nutritionists into the Spanish hospital network would further contribute to the detection, assessment, and treatment of malnutrition, improve the cost-effectiveness ratio in treatment, increase life quality in patients, and achieve a public health support on efficient equal terms.

\section{CITA}

Benítez Brito N. El Dietista-Nutricionista dentro del Sistema Nacional de Salud Español: abordando la desnutrición hospitalaria. Rev Esp Nutr Hum Diet. 2017; 21(2): 199-208. doi: 10.14306/renhyd.21.2.248

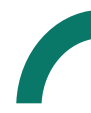

\section{INTRODUCCIÓN}

Han pasado trece años desde que el Comité de Ministros del Consejo de Europa aprobara una serie de medidas encaminadas a estudiar y trabajar en los ámbitos de la alimentación y atención nutricional en los hospitales europeos ${ }^{1}$. Desde entonces, la mayoría de los países han trabajado en el aspecto nutricional, no sólo desde el medio hospitalario, sino también a través de los centros de atención primaria. Sin embargo, no resulta sencillo aplicar estas medidas en los diferentes entornos y, gracias a los equipos multidisciplinarios, se logra avanzar en este aspecto.

En España, los ciudadanos tienen reconocido el derecho a la protección de la salud según La Constitución Española. Esta norma del ordenamiento jurídico establece el reconocimiento universal, con el máximo rango normativo, para el derecho a la protección de salud de todos los españoles (artículo 43)². Por su parte, la Ley 14/1986, de 25 de abril,
General de Sanidad ${ }^{3}$, establece el Consejo Interterritorial del Sistema Nacional de Salud como órgano coordinador entre las Comunidades Autónomas y la Administración General del Estado, de manera que se establece una organización que posibilita la asunción por las Comunidades Autónomas de competencias en materia de sanidad. Estas previsiones constitucionales erigen los principios y criterios que han configurado el Sistema Nacional de Salud (SNS), de forma que permita un funcionamiento cohesionado que garantice el acceso y las prestaciones sanitarias a todos los ciudadanos en condiciones de igualdad efectiva.

España es uno de los países más avanzados en cuanto a materia sanitaria se refiere, aunque en los últimos años la situación ha cambiado. Según el reciente informe Euro Health Consumer Index, que analiza la calidad de los servicios sanitarios de los países europeos, España se sitúa en la posición decimonovena de los treinta y cinco países europeos analizados $^{4}$. Este informe analiza en cada país la cobertura de la cartera de servicios, la accesibilidad, la prevención, la información y los derechos de los pacientes. 


\section{SITUACIÓN ACTUAL DEL DIETISTA-NUTRICIONISTA EN ESPAÑA}

España es el único país de la Unión Europea (UE) que no cuenta con la presencia de Dietistas-Nutricionistas (D-N) en su sistema sanitario en la mayoría de Comunidades Autónomas 5 . Sin embargo, la Ley 44/2003, de 21 de noviembre, de ordenación de las profesiones sanitarias (LOPS), es decir, la ley que regula el ejercicio de las profesiones sanitarias, considera desde el año 2003 al D-N como profesión sanitaria en el SNS 6 . La LOPS considera profesionales sanitarios a los Licenciados en Medicina, Farmacia y Odontología, Diplomados en Enfermería, Fisioterapia, Terapia Ocupacional, Podología, Óptica y Optometría, Logopedia y, finalmente, a los Diplomados en Nutrición Humana y Dietética. Según esta legislación, se considera al D-N como un profesional universitario, especializado en Nutrición Humana y Dietética, que desarrolla actividades orientadas a la alimentación de la persona o grupo de personas, adecuadas a las necesidades fisiológicas y, en su caso, patológicas de las mismas, de acuerdo con los principios de prevención y salud pública ${ }^{6}$.

La Asociación Española de Dietistas-Nutricionistas (AEDN) abogó por la inclusión de los servicios del D-N en el marco de las diferentes áreas del SNS en el año 2009 (Tabla 1) 15,7. En esta publicación se detalla cómo estos profesionales contribuirían en la mejora de la atención sanitaria integrada de los ciudadanos, así como en la optimización de los recursos del propio sistema. Un año más tarde, en el Boletín Oficial de las Cortes Generales del Senado se hace pública la instancia del Senado a reorganizar el SNS sumando otras profesiones sanitarias, entre las que se encuentra precisamente el D-N8.

Actualmente, la presencia de D-N clínicos-hospitalarios dentro del SNS Español es mínima. Las Comunidades Autónomas que cuentan con la categoría profesional de D-N dentro del servicio sanitario de salud son escasas y menos aún son las Comunidades que han podido convocar plazas dentro del grupo B (aunque en realidad debería ser el grupo A2 según la nueva normativa ${ }^{9}$ ) para la titulación en Nutrición Humana y Dietética.

Ahora bien, ¿por qué algunas Comunidades no cuentan con su correspondiente categoría profesional dentro de su autonomía? Principalmente es una cuestión de tiempo, teniendo en cuenta que la titulación en Nutrición Humana y Dietética es muy reciente ${ }^{10}$, sobre todo si se compara con otras profesiones que vienen trabajando ya desde hace varias décadas. Ésta es una limitación que se presenta junto con el proceso tardío de institucionalización de la profesión, ya que hasta ahora eran "otros" los profesionales que realizaban las funciones que le corresponden al D-N, aunque estas funciones no llegan a ser realizadas en la mayoría de las ocasiones ${ }^{11}$.

No obstante, el artículo 15 de la Ley 55/2003, de 16 de diciembre, del Estatuto Marco del Personal Estatutario de los Servicios de Salud ${ }^{12}$, modificado por el Real Decreto-ley 16/2012, de 20 de abril, de medidas urgentes para garantizar la sostenibilidad del SNS y mejorar la calidad y seguridad

Tabla 1. Propuesta de incorporación del Dietista-Nutricionista en el Sistema Nacional de Salud por parte de la Asociación Española de Dietistas-Nutricionistas 5 .

ATENCIÓN PRIMARIA:

1 Dietista-Nutricionista cada 50.000 tarjetas sanitarias.

\section{ATENCIÓN ESPECIALIZADA:}

Unidad U.11. Dietética y Nutrición:

1 Dietista-Nutricionista cada 100 camas en calidad de integrante o responsable de la unidad.

Unidades asistenciales relacionadas con la dietética y nutrición*:

1 Dietista-Nutricionista por unidad asistencial en calidad de integrante.

SALUD PÚBLICA Y PREVENTIVA:

1 Dietista-Nutricionista cada 500.000 habitantes.

* U.6. Alergología, U.7. Cardiología, U.9. Aparato Digestivo, U.10. Endocrinología, U.12. Geriatría, U.14. Nefrología, U.15. Diálisis, U.19. Oncología, U.20. Pediatría, U.69. Psiquiatría y Servicio de alimentación del hospital (restauración hospitalaria). 
de sus prestaciones ${ }^{13}$, establece que cada servicio autonómico creará, modificará o suprimirá las categorías necesarias. Al amparo del citado contexto normativo, así como a la vista de la LOPS ${ }^{6}$, se resalta la necesidad de ir adecuando las categorías profesionales a las necesidades actuales debido a la aparición de nuevos servicios, la evolución de determinadas prestaciones, y la entrada de nuevas titulaciones y especializaciones. En este contexto se hace necesario adaptar a las necesidades y nomenclaturas actuales las determinadas categorías de personal estatutario existentes en el ámbito de las distintas instituciones sanitarias de cada servicio autonómico de salud, de manera que se logre establecer plazas diferenciadas para cada categoría creada, de forma que se regulen las funciones, los requisitos de acceso, y las retribuciones.

En este sentido, recientemente ha sido publicada una nueva actualización según el Real Decreto 184/2015, de 13 de marzo, por el que se regula el catálogo homogéneo de equivalencias de categorías profesionales de personal estatutario de los servicios de salud ${ }^{14}$. Concretamente, en su anexo 1 viene recogido como grupo profesional el D-N con categoría A2, profesional diplomado sanitario.

El presente Real Decreto deja constancia de que el D-N se encuentra enmarcado dentro del SNS, reconociendo por tanto la existencia de esta categoría profesional dentro del propio sistema de salud español ${ }^{14}$.

Sin embargo, aquellas Comunidades que no cuentan con el reconocimiento de su categoría profesional dentro de su autonomía, no pueden apostar por la inclusión del D-N. Sin este requisito no se puede avanzar hacia la introducción progresiva del $\mathrm{D}-\mathrm{N}$, ya que es un requisito indispensable para comenzar a formar parte de la plantilla orgánica de los distintos servicios de salud. Sólo una vez concluida la inclusión de la "categoría profesional D-N" dentro de las categorías profesionales de los diferentes ámbitos sanitarios autonómicos, será una realidad la incorporación progresiva de este profesional en el SNS. En la Tabla 2 se muestran los criterios que deben regir para la creación de dicha categoría.

\section{ABORDAJE DE LA DESNUTRICIÓN HOSPITALARIA: REALIDAD, CIFRAS Y COSTES}

En la actualidad existen pruebas suficientes para integrar a este profesional con titulación expresa en Nutrición Humana y Dietética dentro del SNS ${ }^{5,15-21}$. Los ámbitos posibles de intervención son innumerables y más si se tiene en cuenta

Tabla 2. Criterios que deben regir para la creación de la categoría profesional del Dietista-Nutricionista.

1. Dentro del subgrupo A2 de clasificación se crea la categoría estatutaria de Dietista-Nutricionista, clasificada como personal estatutario sanitario de formación universitaria, en los términos del artículo 6.2.a. 3o de la Ley 55/2003, de 16 de diciembre, del Estatuto Marco del personal estatutario de los servicios de salud.

2. Corresponde al personal Dietista-Nutricionista el desempeño, bajo la dirección del centro sanitario y sin menoscabo de la competencia, responsabilidad y autonomía de los profesionales de otras categorías, de las funciones inherentes a las competencias profesionales especificadas en el programa de formación de la especialidad aprobado de acuerdo con la normativa reguladora de las profesiones sanitarias.

Dichas funciones se desarrollarán preferentemente en los ámbitos asistencial, docente, de gestión clínica, de administración, de investigación, de prevención y de información y educación sanitaria, en relación con los individuos, en grupos de éstos o en familias, tanto en los centros sanitarios como en sus domicilios. Asimismo, se llevarán a cabo participando en aquellas acciones de coordinación socio-sanitaria que a nivel autonómico se determinen.

3. Las retribuciones que percibirá el personal Dietista-Nutricionista se regirán por lo establecido en la Ley 55/2003, de 16 de diciembre, del Estatuto Marco del personal estatutario de los servicios de salud, y su normativa complementaria y de desarrollo, para la categoría profesional de DietistaNutricionista en el correspondiente nivel asistencial. 
la estrecha relación entre enfermedad y nutrición. Este estrecho vínculo ha generado, por ejemplo, nuevos conceptos que hasta ahora no eran tan relevantes para la comunidad sanitaria. En la Tabla 3 se enumera un resumen de las competencias básicas del D-N clínico-hospitalario.

La Desnutrición Relacionada con la Enfermedad (DRE), definida ésta como una alteración que se produce en la composición corporal debido a un déficit de nutrientes (por disminución de la ingesta o aumento de las pérdidas o de los requerimientos) como consecuencia de una enfermedad aguda o crónica, es una condición que disminuye las funciones vitales y repercute negativamente en la evolución clínica de los pacientes, elevando principalmente su morbi- mortalidad ${ }^{22,23}$. Asimismo, son los pacientes desnutridos los que requieren la utilización en mayor medida de los recursos sanitarios, con un mayor número de visitas al médico de atención primaria, estancias hospitalarias más prolongadas, mayor necesidad de centro de convalecencia al alta y mayor porcentaje de reingresos, incrementando a su vez los costes sanitarios ${ }^{15,16,19-21}$. La DRE está haciendo plantear, de nuevo, la necesidad de establecer el estado nutricional dentro de la enfermedad, mostrando así la importancia de los aspectos dietético-nutricionales, teniendo en cuenta que constituye un problema sanitario de elevada prevalencia (afecta a 30 millones de personas en Europa) y altos costes (170.000 millones de euros anuales) $)^{1,22,23}$.

Tabla 3. Resumen de competencias básicas del Dietista-Nutricionista clínico en el área de hospitalización.

Funciones generales:

- Participar de forma general y especializada en la organización, desarrollo y evaluación de las actividades relacionadas con la dietética y la nutrición clínica del hospital.

- Elaborar estándares de actuación profesional en los diferentes ámbitos clínicos asistenciales.

- Participar en los programas de formación continuada.

- Intervenir en las distintas modalidades de soporte nutricional.

- Participar en las comisiones hospitalarias relacionadas con la alimentación, la nutrición y la dietética.

- Supervisar las funciones de los técnicos superiores en dietética.

- Servir de enlace en la coordinación y relación de la Unidad de Nutrición Clínica y Dietética (UNCD) con el servicio de alimentación y la administración del hospital.

Funciones específicas:

- Desarrollar planes de cuidado nutricional para los pacientes identificados con riesgo nutricional o malnutrición.

- Diseñar documentos para la evaluación del estado nutricional, así como protocolos de actuación del soporte nutricional indicado.

- Valorar el estado nutricional del paciente y establecer sus necesidades nutricionales.

- Participar en la elaboración de un plan de intervención individual según su patología y la prescripción nutricional, traduciéndolo a términos alimentarios la prescripción nutricional.

- Planificar el seguimiento y los posibles controles posteriores del soporte nutricional a domicilio, consultas externas o en atención primaria, así como el paso a otras formas de alimentación.

- Intervenir en las sesiones clínicas relativas a la terapia del paciente y colaborar en los trabajos de investigación, así como promover estudios y elaboración de los trabajos de investigación.

- Fomentar la formación continuada en materia de alimentación y nutrición.

- Elaborar informes de forma detallada sobre la dieta o pauta nutricional de alta, y asesorar al paciente y/o familiares.

- Realizar la monitorización y el seguimiento nutricional y dietético del paciente.

- Participar con el servicio de alimentación en la coordinación, en todos los niveles, de las competencias que le son propias, para asegurar la calidad nutricional y gastronómica de la oferta alimentaria, así como velar por las buenas prácticas de higiene en el servicio de alimentación.

- Elaborar y actualizar el manual de dietas hospitalarias.

- Elaborar y actualizar el manual de recomendaciones dietéticas para pacientes al alta hospitalaria.

Perfil de las competencias del titulado universitario en Nutrición Humana y Dietética. Documento de Consenso. Febrero 2003. Disponible en: http://www.consejodietistasnutricionistas.com/wp-content/uploads/2014/06/perfil-completo_DN.pdf 
El Dietista-Nutricionista dentro del Sistema Nacional de Salud Español: abordando la desnutrición hospitalaria

En este sentido, cuando la desnutrición se produce o se diagnóstica en el propio hospital se denomina Desnutrición Hospitalaria $(\mathrm{DH})^{24}$. Los pacientes que ingresan en un hospital son habitualmente el colectivo más susceptible de presentar DRE o riesgo de la misma 24,25 , aunque en general, puede afectar a todos los niveles de atención sanitaria (atención primaria, atención especializada y atención sociosanitaria).

En España, los últimos datos informan de que uno de cada cuatro pacientes hospitalizados está en riesgo de desnutrición o desnutrido $(23,7 \%)$, y que este porcentaje aumenta al $37 \%$ entre los pacientes mayores de 70 años, afectando fundamentalmente a pacientes con enfermedades neoplásicas (35\%), del sistema cardiocirculatorio (29\%) y respiratorio $(28 \%)^{25}$. Además, estudios Ilevados a cabo en pacientes mayores de 65 años muestran una prevalencia de desnutrición del $21,7 \%$ en hospitales, del $15,6 \%$ en residencias, del $2,2 \%$ en sujetos atendidos en atención primaria, y del 0,4\% en los centros de mayores ${ }^{26}$.

A pesar de estos datos, y teniendo en cuenta que son muy similares a los que se encuentran en otros países ${ }^{27}$, en muy pocos hospitales en España se atienden adecuadamente las necesidades nutricionales de los pacientes. En la práctica clínica habitual se cuenta con escaso personal especializado que someta a la totalidad de pacientes a una valoración nutricional, se carece de los suficientes medios para su realización y, finalmente, la codificación de la desnutrición es prácticamente nula en los informes de alta.

Debido a estas preocupantes cifras, la Sociedad Española de Nutrición Enteral y Parenteral (SENPE) ha desarrollado el programa "Masnutridos", mediante el que se buscan medidas de intervención gubernamental para paliar el efecto de la DH. Entre ellas, se ha instado al gobierno central y a los autonómicos a implantar de manera universal un cribado nutricional en todos los hospitales de tercer nivel (hospitales generales) en España. En este sentido, en mayo de 2014 fue aprobado por unanimidad en el Senado el aspecto nutricional como condición específica dentro de las diferentes estrategias que lleva a cabo el Ministerio de Sanidad, Servicios Sociales e Igualdad, y que, conjuntamente con las Comunidades Autónomas y en el marco del Consejo Interterritorial de Salud, acordaron la necesidad de establecer medidas destinadas a la prevención, detección y tratamiento de la desnutrición en todos los ámbitos de la sociedad, con especial atención a pacientes sanitarios y de servicios sociales $^{28}$. Asimismo, determinadas Comunidades Autónomas comienzan a aplicar normativas que impulsen el desarrollo de cribados nutricionales de obligado cumplimiento en el año $2015^{29}$.
No obstante, ya la UE tras el Consejo de Europa sobre alimentación y atención nutricional en hospitales (ResAP 2003), estableció ciertos elementos de obligada consideración ${ }^{1}$. Entiende que la valoración nutricional, la identificación y prevención de las causas de la desnutrición, el soporte nutricional, la alimentación convencional y la alimentación artificial, son cuestiones que deben ser tratadas en todos los marcos hospitalarios y, entre sus recomendaciones en cuanto a la $\mathrm{DH}$, recomendaba la formación universitaria de D-N generales y clínicos a escala nacional, debiendo alcanzar el mayor nivel posible, a fin de permitir que todos los D-N europeos desempeñen un papel relevante en la atención y el soporte nutricional.

Por citar aún más referencias, la European Nutrition for Health Alliance $(E N H A)^{30}$ se fundó con el objetivo de centrar la atención de la desnutrición, su urgencia y su tratamiento. Se proponía como objetivo crear una agenda en la UE y en los países miembros para desarrollar acciones conjuntas e intentar formar una estrategia sanitaria (Together for health: a Strategic Approach for the EU 2007-2013) en consonancia con las líneas de la ResAP 2003‥ En la Declaración de Praga de junio de 200931, los representantes de los Ministerios de Salud de los estados miembros de la Comisión Europea recalcaron de nuevo la necesidad de sistematizar el cribado nutricional obligatorio como uno de los pasos esenciales para combatir la desnutrición en todos los niveles asistenciales. Igualmente, la ENHA, en el año 2014, lanzó otra campaña (Optimal Nutritional Care for All) encaminada a impulsar el desarrollo de programas de cribado nutricional y planes de cuidados nutricionales en ocho países europeos (entre ellos España) ${ }^{32}$.

Pese a la abundante normativa, por no hacer aún más hincapié en la magnitud del problema, resulta ser que la DRE es un problema infravalorado e inadecuadamente tratado, en muchos casos debido al desconocimiento ${ }^{22}$. Hoy en día no está extendida la realización de un cribado nutricional de forma generalizada en todos los niveles asistenciales en España, situación que genera una atención inadecuada de las necesidades nutricionales de muchos pacientes durante su enfermedad $^{33}$. Entre las posibles causas de la inadecuación e infravaloración del tratamiento nutricional se encuentran: en primer lugar, la falta de sensibilización, formación e información de los profesionales sanitarios; en segundo lugar, el desconocimiento sobre la utilización de tratamientos específicos; en tercer lugar, la falta de seguimiento de los pacientes durante su proceso de hospitalización, así como entre los distintos niveles de atención sanitaria; y por último, la falta de inclusión de nuevos profesionales con titulación expresa en Nutrición Humana y Dietética dentro del SNS1,11,33. 
El Dietista-Nutricionista dentro del Sistema Nacional de Salud Español: abordando la desnutrición hospitalaria

Existiendo grandes grupos de trabajo, alianzas, organismos nacionales e internacionales, sociedades científicas, entre otras, vuelve a resultar paradójico cómo, tendiendo en la actualidad dificultades para llevar a cabo una adecuada intervención nutricional en los hospitales de tercer nivel en España, no se preste la importancia debida a un profesional expresamente cualificado en esta materia como es el caso del D-N. Como se ha citado anteriormente, en el año 2009 la entonces $A E D N$, reconvertida hoy en día en la Academia Española de Nutrición y Dietética, ofreció una propuesta para la incorporación del D-N en el SNS de manera progresiva (Tabla 1$)^{5}$, a raíz de los datos que planteaba la UE y la Organización Mundial de la Salud (OMS) ${ }^{34,35}$. Sin embargo, lamentablemente esta situación no se ha convertido en realidad y parece que la situación no tiene visos de mejora a corto plazo.

Según el Real Decreto 1277/2003, de 10 de octubre, que constituye las bases generales sobre autorización de centros, servicios y establecimientos sanitarios, las Unidades de Nutrición y Dietética se definen como unidades asistenciales encargadas de la adecuada nutrición de los pacientes ingresados y de todos aquellos que precisen continuar con tratamiento tras el ingreso hospitalario ${ }^{36}$. Inicialmente, en 1997, se negoció una Cartera de Servicios de Endocrinología y Nutrición entre expertos miembros de la Sociedad Española de Endocrinología y Nutrición (SEEN) y el Instituto Nacional de la Salud, que posteriormente ha sido actualizada en el año 2011 y, recientemente, publicada por la SEEN en este mismo año. No obstante, ya desde el año 2011 se considera fundamental la inclusión del D-N dentro de la cartera de servicios de esta área por parte de la SEEN, con funciones específicas dentro de un grupo multidisciplinar que incorpora varios profesionales ${ }^{37-39}$.

La incorporación del D-N al SNS, especialmente en atención primaria y especializada, ofrecería la posibilidad final de combatir la DRE en todos sus niveles, pero además, ofrecería seguridad y eficacia en los servicios relacionados con la atención nutricional. Y no sólo contribuiría a la mejora de la salud, sino que serviría incluso para optimizar las inversiones en sanidad mejorando así la relación coste-efectividad ${ }^{5,7}$.

El pasado año 2016 se inició la elaboración de un documento marco a nivel nacional para el abordaje de la DRE en el SNS que continúa en fase de borrador y pendiente de ser publicado. Este documento ha sido elaborado mediante consenso entre un grupo multidisciplinar, con representantes de sociedades científicas y profesionales expertos vinculadas al abordaje de la DRE, que han contribuido a la identificación de puntos críticos y necesidades en la atención relacionada con esta circunstancia de salud. En las estrategias de salud del SNS que Ilevan a cabo los expertos, se establecen un conjunto de objetivos y recomendaciones que permiten orientar la organización de los servicios hacia la mejora de la salud de la población y sus determinantes, la prevención de las condiciones de salud y las limitaciones en la actividad. A lo largo de estos últimos años se han ido identificado diferentes proyectos, estrategias y líneas de trabajo para llevar a cabo la implementación, el seguimiento y la evaluación de las mismas.

Es evidente que este manifiesto es una auténtica oportunidad para el colectivo de $\mathrm{D}-\mathrm{N}$, principalmente porque si se detallara la incorporación de este profesional dentro del grupo de trabajo, abogando por su inclusión dentro del SNS, se generaría un sistema sanitario de acuerdo a los estándares propuestos por la UE y la $\mathrm{OMS}^{34,35}$. En este aspecto, son varios los D-N que se han especializado y trabajan en las premisas sobre la DRE, por lo que una vez más no se entendería la reticencia a incorporar esta figura profesional ${ }^{40-44}$. En este sentido, el Consejo General de Dietistas-Nutricionistas de España deberá abogar aún más por la inclusión de este profesional en el desarrollo de los planes de actuación de forma que se configuren equipos donde se integre al D-N.

Aunque el aspecto más relevante a la hora de abordar la $\mathrm{DH}$ es la aplicación de un cribado nutricional que permita detectar al paciente con peor pronóstico clínico (fundamentalmente en base a parámetros nutricionales) a fin de dar un tratamiento nutricional que pueda mejorar dicho pronóstico, se hace necesario, en vistas de introducir al D-N hospitalario en el SNS, aportar datos económicos para consolidar la implementación de cualquier tipo de medida de prevención sanitaria.

Diversos trabajos han mostrado el aumento del coste económico asociado a la DH debido fundamentalmente a la prolongación de la estancia hospitalaria, aumento de reingresos y al tratamiento adicional de las complicaciones ${ }^{19-21,40,45,46}$. En Reino Unido, por ejemplo, el Instituto Nacional para Salud y Excelencia Clínica (NICE, por sus siglas en inglés) publicó una lista de ahorros de costes para apoyar su sistema sanitario, y extensible a otros estados para que puedan compartir esta visión en la toma de conciencia con determinadas medidas terapéuticas. Si se mejorara la atención nutricional de los pacientes mediante la implantación de un cribado universal y se llevara a cabo una correcta evaluación con su respectivo tratamiento, se conseguiría tener pacientes mejor nutridos con una clara reducción de las complicaciones hospitalarias más prevalentes. Las estimaciones más conservadoras de la menor cantidad de ingresos y reducción de la permanencia de los pacientes ingresados, la reducción de la demanda de atención por el médico de cabecera y otras consultas externas, indican un importante ahorro que podría ascender a 28.472 libras por 100.000 
habitantes ${ }^{47}$. Por su parte, Países Bajos, país que cuenta con bastante experiencia en el ámbito de la $\mathrm{DH}$, mostró cómo la intervención nutricional (a través de la implementación de cribados nutricionales) permitía identificar a los pacientes en riesgo nutricional, reduciendo la estancia hospitalaria en un día y, por tanto, reduciendo el coste por hospitalización ${ }^{19,20}$. El beneficio que se obtiene cuando se trata adecuadamente la DRE supone un ahorro neto de entre $1.433 \mathrm{y}$ 3.105 euros por paciente. Las estimaciones que de nuevo realizaban los neerlandeses son que por cada euro invertido en el tratamiento de la persona con DRE la sociedad ahorra entre 1,9 y 4,2 euros, mostrando por tanto que el tratamiento de la DRE es coste-efectivo ${ }^{21}$. Además, sus conclusiones van más allá en términos de coste anual, ya que si se tratara a la práctica totalidad de los pacientes desnutridos con cánceres gastrointestinales y de pulmón, cáncer de cabeza y cuello, y pacientes ancianos hospitalizados, se ahorraría entre 4-42 millones, 1,5-3,8 millones, y 15-78 millones de euros anuales, respectivamente ${ }^{21}$.

En España, los datos económicos en relación a la $\mathrm{DH}$ se han obtenido gracias al estudio Predyces ${ }^{\circledR 25}$. Los resultados de este estudio estiman un mayor coste hospitalario por parte de los pacientes desnutridos respecto a los normonutridos (8.590 euros frente a 7.085 euros) con una diferencia media de 1.505 euros. Además, el coste que se le atribuye a la DH en España ronda los 1.143 millones de euros, suponiendo este coste aproximadamente el $2 \%$ del presupuesto del SNS25.

\section{$\longrightarrow$ \\ CONCLUSIONES}

La identificación precoz de los pacientes que presentan $\mathrm{DH}$, y la pronta actuación mediante tratamiento nutricional, reduciría costes económicos en base fundamentalmente a su efecto atenuante sobre ciertos parámetros (estancia media, reingreso y complicaciones). En este sentido, la introducción progresiva de un D-N por cada hospital de tercer nivel en España es un propósito tibio para abordar la $\mathrm{DH}$, pero sin embargo, supondría un ahorro de costes en términos hospitalarios y sería un gran paso de cara a la incorporación del colectivo D-N en el ámbito clínico. Un D-N por centro hospitalario con dedicación exclusiva al cribado nutricional, en conjunto con un equipo multidisciplinar, sería una forma coherente de actuar en consonancia contra la DRE en España. De este modo, se detectaría la desnutrición en el momento del ingreso hospitalario, se procedería a la valoración nutricional y se intervendría en conjunto con el equipo médico en la intervención nutricional. En consecuencia, España cumpliría además con los estándares propuestos por la UE y la OMS.

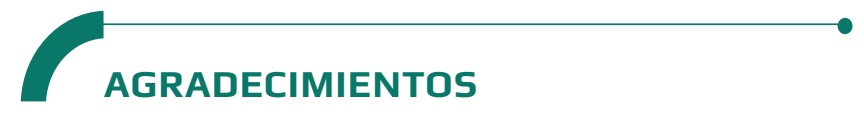

Agradecer las sugerencias y comentarios de D. Andrés Alcántara Escobar y Dña. Cristina Luján López. Igualmente dar las gracias por sus opiniones al Dr. Enrique Palacio Abizanda y a la Dra. Francisca Pereyra-García Castro. Finalmente, agradecer a todos y a cada uno de los dietistas-nutricionistas que contribuyen diariamente con su labor al reconocimiento de la profesión en España.

\section{Conflicto de intereses}

El autor expresa como conflicto de interés ser Dietista-Nutricionista, miembro del cuerpo de académicos de la Academia Española de Nutrición y Dietética, miembro de la Sociedad Española de Nutrición Enteral y Parenteral, y vicepresidente de la Asociación Canaria de Dietistas-Nutricionistas.

\section{REFERENCIAS}

(1) Council of Europe. Resolution ResAP(2003)3 on food and nutritional care in hospitals. Bruselas, Bélgica: Committee of Ministers; 2003.

(2) Cortes Generales. Constitución Española. BOE núm. 311, 1978. Sec. I. Disposiciones generales, 31229 p. 29313-424.

(3) Jefatura del Estado. Ley 14/1986, de 25 de abril, General de Sanidad. BOE núm. 102, 1986. Sec. I. Disposiciones generales, 10499 p. 15207-24.

(4) Björnberg A. Euro Health Consumer Index 2015 Report. Marseillan, Francia: Health Consumer Powerhouse; 2016.

(5) Russolillo G, Baladia E, Moñino M, Colomer M, García M, Basulto J, et al. Incorporación del dietista-nutricionista en el Sistema Nacional de Salud (SNS): Declaración de Postura de la Asociación Española de Dietistas-Nutricionistas (AEDN). Act Diet. 2009; 13(2): 62-9.

(6) Jefatura del Estado. Ley 44/2003, de 21 de noviembre, de ordenación de las profesiones sanitarias. BOE núm. 280, 2003. Sec. I. Disposiciones generales, 21340 p. 41442-58.

(7) Baladia E, Basulto J, Padró L. Documento para la introducción del dietista-nutricionista en el Sistema Sanitario Catalán. Act Diet. 2008; 12(1): 20-6.

(8) Russolillo G. Comparecencia del Presidente de la Asociación Española de Dietistas y Nutricionistas, D. Giuseppe Russolillo Femenías. Sec. Cortes Generales, 483 Madrid, España: Boletín Oficial de las Cortes Generales; 2010 p. 21.

(9) Ministerio de Sanidad, Servicios Sociales e Igualdad. Real Decreto 184/2015, de 13 de marzo, por el que se regula el catálogo homogéneo de equivalencias de las categorías profesionales del personal estatutario de los servicios de salud y el procedimiento de su actualización. BOE núm. 83, 2015. Sec. I. Disposiciones generales, 3717 p. 29447-61. 
(10) Ministerio de Educación y Cultura. Real Decreto 433/1998, de 20 de marzo, por el que se establece el título universitario oficial de Diplomado en Nutrición Humana y Dietética y las directrices generales propias de los planes de estudios conducentes a la obtención de aquél. BOE núm. 90, 1998. Sec. I. Disposiciones generales, 8914 p. 12439-41.

(11) Trescastro-López EM. El papel del movimiento sanitario internacional en el desarrollo de la profesión de DietistaNutricionista en España. Rev Esp Nutr Hum Diet. 2015; 19(1): 49-55.

(12) Jefatura del Estado. Ley 55/2003, de 16 de diciembre, del Estatuto Marco del personal estatutario de los servicios de salud. BOE núm. 301, 2003. Sec. I. Disposiciones generales, 23101 p. 44742-63.

(13) Jefatura del Estado. Real Decreto-ley 16/2012, de 20 de abril, de medidas urgentes para garantizar la sostenibilidad del Sistema Nacional de Salud y mejorar la calidad y seguridad de sus prestaciones. BOE» núm. 98, 2012, 5403 p. 31278-312.

(14) Ministerio de Sanidad, Servicios Sociales e Igualdad. Real Decreto 184/2015, de 13 de marzo, por el que se regula el catálogo homogéneo de equivalencias de las categorías profesionales del personal estatutario de los servicios de salud y el procedimiento de su actualización. BOE núm. 83, 2015. Sec. I. Disposiciones generales, 3717 p. 29447-61.

(15) Hampl JS, Anderson JV, Mullis R, American Dietetic Association. Position of the American Dietetic Association: the role of dietetics professionals in health promotion and disease prevention. J Am Diet Assoc. 2002; 102(11): 1680-7.

(16) Carey M, Gillespie S. Position of The American Dietetic Association: cost-effectiveness of medical nutrition therapy. J Am Diet Assoc. 1995; 95(1): 88-91.

(17) Baladia E, Basulto J. El dietista-nutricionista en atención primaria de salud. Grupo de Revisión y Posicionamiento de la Asociación Española de Dietistas-Nutricionistas (GREP/ AED-N); 2005.

(18) Howatson A, Wall CR, Turner-Benny P. The contribution of dietitians to the primary health care workforce. J Prim Health Care. 2015; 7(4): 324-32.

(19) Kruizenga $H$, van Keeken S, Weijs P, Bastiaanse L, Beijer S, Huisman-de Waal $G$, et al. Undernutrition screening survey in 564,063 patients: patients with a positive undernutrition screening score stay in hospital $1.4 \mathrm{~d}$ longer. Am J Clin Nutr. 2016; 103(4): 1026-32.

(20) Kruizenga HM, Van Tulder MW, Seidell JC, Thijs A, Ader HJ, Van Bokhorst-de van der Schueren MAE. Effectiveness and costeffectiveness of early screening and treatment of malnourished patients. Am J Clin Nutr. 2005; 82(5): 1082-9.

(21) Kok L, Scholte R. Malnutrition underestimated: The costs of malnutrition and the return on medical nutrition. SEO Economic Research; 2014.

(22) Ljungqvist O, Man F de. Under nutrition: a major health problem in Europe. Nutr Hosp. 2009; 24(3): 369-70.

(23) Stratton RJ, Green C], Elia M. Consequences of Disease-related Malnutrition. En: Disease-related Malnutrition: An Evidencebased Approach to Treatment. Wallingford, UK: CABI Pub.; 2003. p. 113-55.

(24) García A, Álvarez J, Planas M, Burgos R, Araujo K, multidisciplinary consensus work-team on the approach to hospital malnutrition in Spain. Multidisciplinary consensus on the approach to hospital malnutrition in Spain. Nutr Hosp. 2011; 26(4): 701-10.

(25) Álvarez-Hernández J, Planas M, León-Sanz M, García de Lorenzo A, Celaya-Pérez S, García-Lorda P, et al. Prevalence and costs of malnutrition in hospitalized patients; the PREDyCES Study. Nutr Hosp. 2012; 27(4): 1049-59.

(26) Cuerda C, Álvarez J, Ramos P, Abánades JC, García-de-Lorenzo $A$, Gil P, et al. Prevalencia de desnutrición en sujetos mayores de 65 años en la Comunidad de Madrid. Estudio DREAM + 65. Nutr Hosp. 2016; 33(2): 101.

(27) Sorensen J, Kondrup J, Prokopowicz J, Schiesser M, Krähenbühl L, Meier R, et al. EuroOOPS: an international, multicentre study to implement nutritional risk screening and evaluate clinical outcome. Clin Nutr. 2008; 27(3): 340-9.

(28) Comisión de Sanidad y Servicios Sociales. Mociones en Comisión del Grupo Parlamentario Popular en el Senado, por la que se insta al gobierno a la adopción de determinadas medidas para la lucha contra la desnutrición. Sec. Cortes Generales, 341 Madrid, España: Boletín Oficial de las Cortes Generales; 2014 p. 8-9.

(29) Parlamento de Canarias. Proposición No de Ley 8L/PNL0326 Sobre tratamiento de la desnutrición. Boletín Oficial del Parlamento de Canarias núm. 227, 2014. Sec. Resoluciones Aprobadas. Enmiendas p. 5.

(30) The European Nutrition for Health Alliance. STOP diseaserelated malnutrition and diseases due to malnutrition! Final Declaration. Čestlice, República Checa: The European Nutrition for Health Alliance; 2009.

(31) European Nutrition for Health Alliance, The European Society for Clinical Nutrition and Metabolism, Medical Nutrition International Industries, Association Internationale de la Mutualité, European Hospital and Healthcare Federation, European Nursing Directors Association, et al. The Prague Declaration: A call for action to fight malnutrition in Europe. 2007.

(32) European Nutrition for Health Alliance. A World with Optimal Nutritional Care for All: Report of the 1st Optimal Nutritional Care for All Conference 4 th /5 th November 2014, Brussels. Bruselas, Bélgica: European Nutrition for Health Alliance; 2014.

(33) Martín T. Gestión en Nutrición Clínica. Nutr Hosp. 2015; 31(Suppl 5): 5-15.

(34) Ministère de la santé (France). Alimentation en milieu hospitalier : rapport de mission à Monsieur le Ministre chargé de la santé. Lassay-les-Châteaux, Francia: Secrétariat d’Etat à la santé et à la sécurité sociale; 1995.

(35) Organización Mundial de la Salud (OMS): 10.a Asamblea Mundial de la Salud 1974; 29.a Asamblea Mundial de la Salud 1976, resolución WHA29.72; 35 Asamblea Mundial de la Salud 1982, resolución WHA35.25. 1982.

(36) Ministerio de Sanidad y Consumo. Real Decreto 1277/2003, de 10 de octubre, por el que se establecen las bases generales sobre autorización de centros, servicios y establecimientos sanitarios. BOE núm. 254, 2003. Sec. I. Disposiciones generales, 19572 p. 37893-902.

(37) Monereo S, Barceló B, López J, Marco Mur A, Marañés JP, Pallardo LF, et al. Cartera de servicios de endocrinología y nutrición. Endocrinol Nutr. 1999; 46(6): 180. 
(38) Vicente A, Gómez P, Tinahones F. Cartera de Servicios de Endocrinología y Nutrición. Endocrinol Nutr. 2011; 58(3): 127-42.

(39) Navarro E, Herrero A, Moreno A, Brandes O, Murillo J, Ballesteros MD. Cartera de servicios en Endocrinologia y Nutricion 2016. SEEN; 2016.

(40) Pérez A, Lobo G, Orduña R, Mellado C, Aguayo E, Ruiz MD. Desnutrición en pacientes hospitalizados: prevalencia e impacto económico. Med Clin. 2004; 123(6): 201-6.

(41) Calleja A, Vidal A, Cano I, Ballesteros MD. Eficacia y efectividad de las distintas herramientas de cribado nutricional en un hospital de tercer nivel. Nutr Hosp. 2015; 31(5): 2240-6.

(42) Oliva JG, Pereyra-García F, Benítez N, Herrera EM, Suárez JP, García BF, et al. Validación de un método de dispensación de suplementos nutricionales en un hospital terciario. Nutr Hosp. 2013; 28(4): 1286-90.

(43) Suárez JP, Benitez N, Oliva JG, Pereyra-García F, López MA, García $A$, et al. Introducción de un método mixto de cribado nutricional (CIPA) en un hospital de tercer nivel. Nutr Hosp. 2014; 29(5): 1149-53.
(44) Benítez N, Mora A, Suárez JP, Delgado I, Pérez LI, Herrera EM, et al. Concordancia en los resultados tras la realización de un control de ingestas de $72 \mathrm{~h}$ por diferente personal sanitario en un hospital de tercer nivel. Nutr Hosp. 2015; 32(6): 2893-7.

(45) Freijer K, Tan SS, Koopmanschap MA, Meijers JMM, Halfens RJG, Nuijten MJC. The economic costs of disease related malnutrition. Clin Nutr. 2013; 32(1): 136-41.

(46) Correia MITD, Waitzberg DL. The impact of malnutrition on morbidity, mortality, length of hospital stay and costs evaluated through a multivariate model analysis. Clin Nutr. 2003; 22(3): 235-9.

(47) NICE. Clinical and Cost Effective Prescribing of Oral Nutritional Supplements for Adults in the Community [Internet]. 2011 [citado 20 de agosto de 2016]. Disponible en: https://www. nice.org.uk/sharedlearning/clinical-and-cost-effectiveprescribing-of-oral-nutritional-supplements-for-adults-in-thecommunity. 